\title{
Properties Evaluation and Application of Organic Amine Inhibitor on the Properties of Drilling Fluids
}

\author{
Kaihe Lv*, Hanyi Zhong, Guanlong Ren and Yuxia Liu \\ School of Petroleum Engineering, China University of Petroleum, Qingdao, 266580, China
}

\begin{abstract}
An organic amine inhibitor was developed and evaluated. Experimental results showed that the organic amine inhibitor could decrease the surface tension to $50 \mathrm{mN} / \mathrm{m}$ and had a little effect on the Zeta potential of clay. The inhibitor could effectively inhibit the dispersion of core and cuttings and had a lasting strong inhibition. The inhibitor had good compatibility with common drilling fluid, and it was useful to maintain drilling fluid stability under high temperature and could improve the filtration property of drilling fluid.
\end{abstract}

Keywords: Drilling fluids, inhibitive properties, mechanism, organic amine inhibitor.

\section{INTRODUCTION}

In order to meet the requirements of environmental protection and reduce drilling cost, people at home and abroad are in search for a kind of water-based drilling fluid with the performance of oil-based drilling fluid [1-4], and have adopted various methods to improve the performance of water-based drilling fluid, such as adding inorganic or organic salt, cationic polymer and polymeric alcohol etc. However, these drilling fluids have many kinds of limitations in inhibiting shale swelling and hydration [5-7]. In recent years, a novel type of high performance water-based drilling fluid system is introduced which is mainly composed of organic amine shale hydration inhibitor, cationic polyacrylamide encapsulator, rheology modifier, and lubricant and filtration reducer. The system has obtained wide application around the world with great success because of excellent inhibition, lubrication and stable rheology and so on. As the key additive, organic amine has higher inhibitory and anti-balling abilities. It can be used in various kinds of water-based drilling fluids and has a very good compatibility with them. It can meet environmental protection requirements and has film-forming effect, and the performance of high performance water-based drilling fluid made by it is almost the same as that made with oil-base drilling fluid system [8-11]. This paper developed an organic amine inhibitor with good comprehensive performance and its performance is evaluated.

\section{PREPARATION OF ORGANIC AMINE INHIBITOR}

Initiator and alkali catalyst are added to the reaction kettle with heating vacuum dehydration, then epoxy ethane or epoxy propane is pressured into the reaction kettle. When the pressure in the reaction kettle does not drop, the reaction is over. The intermediate product is yielded after cooling, filtering and refining. Then the intermediate product, catalyst, low-molecular amine and hydrogen are added to the high

*Address correspondence to this author at the School of Petroleum Engineering, China University of Petroleum, Qingdao, 266580, China; Tel: 0086-546-8392274; Fax: 0086-546-8394600;

Email:1kh54321@126.com pressure reaction kettle, after reaction for a period of time at a certain temperature, cooling, filtering and refining, the organic amine inhibitor is obtained.

\section{EFFECTS OF ORGANIC AMINE INHIBITOR ON THE PROPERTIES OF DRILLING FLUIDS}

\subsection{Effect on Surface Tension of Drilling Fluids}

Surface tension method is used to test the surface activity of organic amine inhibitor. Fig. (1) shows that in a very low concentration of organic amine, the surface tension of organic amine aqueous solution decreases sharply. Along with the concentration increase, the drop speed of surface tension becomes low. In the surface tension curve $(\gamma-C)$, it is generally considered that the concentration at the inflection point is the critical micelle concentration (CMC) of surface active material. According to the tangent to $\gamma-\mathrm{C}$ curve, $\mathrm{CMC}$ of organic amine is about $0.21 \mathrm{wt} \%$. Organic amine can decrease the surface tension to $50 \mathrm{mN} / \mathrm{m}$, which is beneficial to reduce the water block damage of low permeability reservoir caused by drilling fluids $[12,13]$.

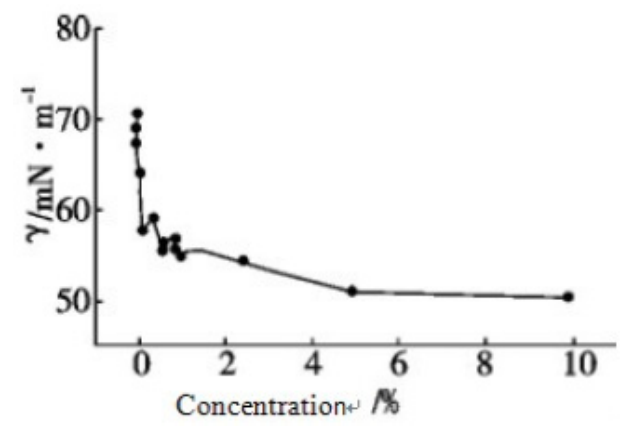

Fig. (1). Surface tension curve of organic amine aqueous solution.

\subsection{Effect on Zeta Potential of Clay Particles}

Variations of Zeta potential of the bentonite particles after adsorption equilibrium with the concentration of organic amine inhibitor were measured. Before adding organic amine 
inhibitor, the Zeta potential of the bentonite particles was $40 \mathrm{mV}$, indicating a typical sodium bentonite. Within the experiment concentration range $(0.1 \% \sim 1 \%)$, as the concentration increased, the absolute value of Zeta potential of clay particles decreased a little, about $-40 \mathrm{mV} \sim-35 \mathrm{mV}$, and the overall changes were not big. It does not appear whether electronic charge reversal in sign due to typical cationic surfactant adsorbs on clay particles. It is expected that organic amine inhibitor has a certain positive charge and can be absorbed on drilling cuttings or surface of borehole wall which is beneficial to borehole wall stability, but the capacity of electricity is not enough to damage the stability of the clay dispersion system [14, 15] Fig. (2) shows that Zeta potential of the bentonite particles varies after adsorption equilibrium with the concentration of organic amine inhibitor.

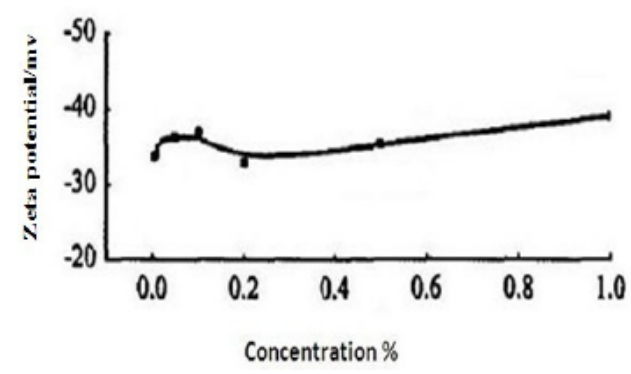

Fig. (2). Variations of Zeta potential of the bentonite particles with different concentration of organic amine inhibitor.

\section{EFFECT ON INHIBITION PERFORMANCE OF DRILLING FLUIDS}

\subsection{Effect on the Slurry Properties of Clay}

Inhibition on mud making and maintenance on drilling fluid rheological property are the most applicable methods to evaluate shale inhibitors. A certain amount of bentonite was added to the distilled water, under stirring for $20 \mathrm{~min}$ as the base slurry; then $2 \%$ organic amine inhibitor was added to the slurry as sample slurry. Base slurry and sample slurry were placed in each high-temperature jar and hot-rolled for 16 hours in the roller with the fluid circulating at $65^{\circ} \mathrm{C}$. Afterwards, the jars with fluid were cooled down to room temperature and its rheological property was measured. It was continued to increase the content of bentonite and repeat the above experiment process. The experimental results are shown in (Table 1).

As can be seen from Table 1, when the bentonite contents are same, with increasing of organic amine inhibitor added, the viscosity and shearing force of the drilling fluids obviously lower than that without inhibitors, indicating that organic amine inhibitor can effectively restrain the bentonite slurry.

\subsection{Evaluation on Inhibiting Dispersion Performance of Cuttings}

Using the experiment of shale hot-rolling recovery, the inhibiting dispersion performance of cuttings was evaluated, and the experimental results are shown in (Table $\mathbf{2}$ and Table 3). The cuttings were derived from Shengli Oilfield and Xinjiang Oilfield respectively and the experiment procedures were: $50.0 \mathrm{~g}$ (weighing rightly to $0.1 \mathrm{~g}$ ) shale sample with particles diameter of 2-5 $\mathrm{mm}$ was weighed and put into scroll bottle filled with $350 \mathrm{~mL}$ soak solution and covered closely, and was then put into roller heating furnace at the temperature of $77 \pm 1^{\circ} \mathrm{C}$. After rolling for 16 hours, it was cooled down to room temperature and poured into the $0.45 \mathrm{~mm}$ standard sieve, and it was rinsed with clear water for one minute; and then the remains of shale sample were put into drying oven at $105 \pm 3{ }^{\circ} \mathrm{C}$ of constant temperature for 4 hours. After taking it out, it was left in the air for 24 hours, and then the quantity was weighed. Recovery rate of shale which was calculated by the quantity of shale remained same as the quantity of original shale sample.

Table 1. Mud making performance of bentonite in different mediums.

\begin{tabular}{|c|c|c|c|c|}
\hline $\begin{array}{c}\text { Amount of } \\
\text { organic } \\
\text { amine/\% }\end{array}$ & $\begin{array}{c}\text { Amount of } \\
\text { bentonite }\end{array}$ & $\begin{array}{c}\text { AV } \\
/ \mathbf{m P a} \cdot \mathbf{s}\end{array}$ & $\begin{array}{c}\mathbf{P V} \\
/ \mathbf{m P a} \cdot \mathbf{s}\end{array}$ & $\begin{array}{c}\mathbf{Y P} \\
/ \mathbf{P a}\end{array}$ \\
\hline \hline \multirow{2}{*}{0} & $3 \%$ & 5 & 3 & 2 \\
\cline { 2 - 5 } & $6 \%$ & 20 & 8 & 12 \\
\cline { 2 - 5 } & $9 \%$ & 75.5 & 5 & 70.5 \\
\hline \multirow{2}{*}{2} & $3 \%$ & 2 & 2 & 0 \\
\cline { 2 - 5 } & $3 \%$ & 10.5 & 10 & 0.5 \\
\cline { 2 - 5 } & $3 \%$ & 15.5 & 8 & 7.5 \\
\hline
\end{tabular}

As can be seen from Table $\mathbf{2}$ and Table $\mathbf{3}$, the recovery ratio of organic amine aqueous solution is much higher than $7 \% \mathrm{KCl}$ and $3 \% \mathrm{NaSiO}_{4}$, especially the second and the third recovery ratio is higher, indicating that organic amine can strongly adsorb on the surface of cuttings and has good longterm inhibitory activity, and can effectively inhibit hydration dispersing effect of the shale cuttings and be helpful to borehole wall stability and reservoir protection.

Table 2. Dispersion and slurry performance of cuttings in different medium.

\begin{tabular}{|c|c|c|c|}
\hline \multirow{2}{*}{ Soak solution } & \multicolumn{3}{|c|}{ Recovery ratio/\% } \\
\cline { 2 - 4 } & First & Second & Third time \\
\hline \hline Water & 39.82 & 17.26 & 9.56 \\
\hline $2 \%$ organic amine & 82.06 & 73.62 & 70.30 \\
\hline $7 \% \mathrm{KCl}$ & 68.00 & 36.84 & 20.22 \\
\hline $3 \% \mathrm{NaSiO}_{4}$ & 60.52 & 39.62 & 18.98 \\
\hline
\end{tabular}

* The cuttings are from the Shengli Oilfield

\subsection{Evaluation on Cores Soaking Experiment}

By observing the complete degree of the artificial cores soaked at different solutions after a certain time, the inhibition ability of different treating chemicals can be compared qualitatively. Preparation of the cores: $20 \mathrm{~g}$ bentonite was weighed and put into core mould; the mould was suppressed at pressure of $15 \mathrm{MPa}$ for 10 minutes then the core was taken out carefully for reserve. Core was soaked in different solu- 
tions and its changes were observed. After being soaked for $2 \mathrm{~h}$, core dispersed and collapsed completely in the solutions of $7 \% \mathrm{KCl}, 10 \% \mathrm{NaCl}$ and $5 \% \mathrm{NaSiO}_{4}$ but there was only some spalling on the edge of the core soaked in organic amine aqueous solution as Fig. (3) shows. The core remained basically integrated, indicating organic amine inhibitor can effectively inhibit the dispersion of core.

Table 3. Dispersion and slurry performance of cuttings in different medium.

\begin{tabular}{|c|c|c|c|}
\hline \multirow{2}{*}{ Soak solution } & \multicolumn{3}{|c|}{ Recovery ratio/\% } \\
\cline { 2 - 4 } & First & Second & Third time \\
\hline \hline Water & 36.62 & 15.28 & 8.76 \\
\hline $2 \%$ organic amine & 91.62 & 80.33 & 73.23 \\
\hline $7 \% \mathrm{KCl}$ & 65.33 & 52.62 & 40.23 \\
\hline $3 \% \mathrm{NaSiO}_{4}$ & 55.32 & 31.23 & 21.56 \\
\hline
\end{tabular}

* The cuttings are from the Xinjiang Oilfield

\subsection{Effect on Rheological Property and Filtration of Drilling Fluids}

A good kind of inhibitor can not only improve inhibition performance but should also be devoid of adverse impact on other performance of the drilling fluids, therefore the effect of organic amine inhibitors on the conventional performance of the drilling fluids was evaluated. Different amounts of organic amine inhibitor were added to the $4 \%$ prehydrated bentonite mud and their rheological property and filtration were tested before and after high temperature, and the results are shown in (Table 4).

As can be seen from Table 4 , within the $2 \%$ range of organic amine inhibitor, the rheological parameters of the drilling fluids after hot rolling for 16 hours are close to those before hot rolling and there does not appear significant reduction or rise, indicating organic amine inhibitor will not affect the basic rheological properties and high temperature stability performance of the drilling fluids. With the increase of added organic amine, the filtration declines from $32.8 \mathrm{~mL}$ to $25.4 \mathrm{~mL}$ and after hot rolling for 16 hours it declines from $36.6 \mathrm{~mL}$ to $28.0 \mathrm{~mL}$ which shows organic amine inhibitor has some de-filtration effect.

\section{MECHANISM ANALYSIS OF ORGANIC AMINE INHIBITOR}

\subsection{Charge Neutralization}

After organic amine inhibitor was dissolved in water, a certain amount of positive charge adsorbed to the clay particles through the adsorption group or electrostatic interaction, and it can neutralize the negative charge on the surface of the clay and make hydration expansion and dispersion of the clay weakened which is helpful to the stability of borehole wall.

\subsection{Adsorption}

Hydroxyl and other absorbing groups of the molecular on the organic amine inhibitor can be adsorbed onto the surface of the borehole wall by absorption and electrostatic interaction, and it can form a hydrophobic adsorption layer on the surface of the borehole wall and prevent wall rock to contact with drilling fluids and is advantageous to the stability of borehole wall.

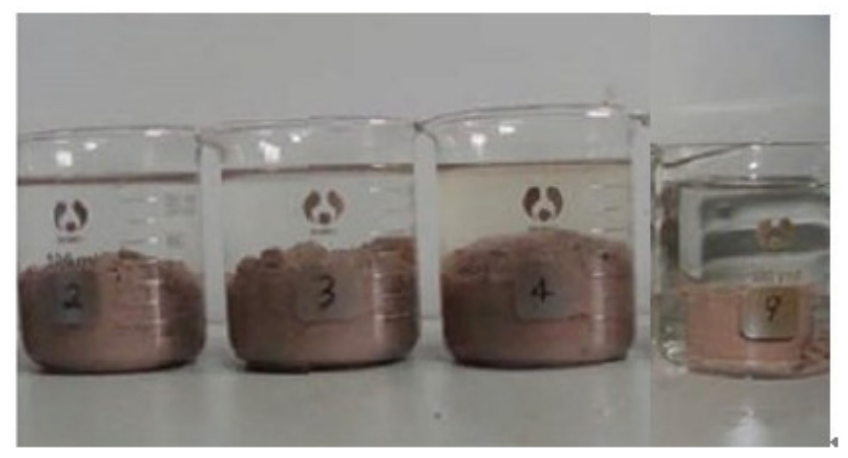

Fig. (3). The changes of shale sample after soaking in various solutions for $2 \mathrm{~h}$

Table 4. Effect of organic amine inhibitor on the property of drilling fluids.

\begin{tabular}{|c|c|c|c|c|c|}
\hline Amount & $\underset{/ \mathbf{m P a} \cdot \mathbf{s}}{\mathbf{A V}}$ & $\begin{array}{c}\text { PV } \\
/ \mathbf{m P a} \cdot \mathbf{s}\end{array}$ & $\begin{array}{l}\mathbf{Y P} \\
/ \mathbf{P a}\end{array}$ & $\begin{array}{c}\text { API FL } \\
/ / \mathbf{m l}\end{array}$ & Remarks \\
\hline \multirow{2}{*}{0} & 12.5 & 5 & 7.5 & 32.8 & Room temperature \\
\hline & 6 & 5 & 1 & 36.6 & $120^{\circ} \mathrm{C} / 16 \mathrm{~h}$ \\
\hline \multirow{2}{*}{$0.5 \%$} & 13.5 & 6 & 7.5 & 24.0 & Room temperature \\
\hline & 10 & 6 & 4 & 28.0 & $120^{\circ} \mathrm{C} / 16 \mathrm{~h}$ \\
\hline \multirow{2}{*}{$1.0 \%$} & 13.5 & 6 & 7.5 & 24.6 & Room temperature \\
\hline & 10.5 & 6 & 4.5 & 27.4 & $120^{\circ} \mathrm{C} / 16 \mathrm{~h}$ \\
\hline \multirow{2}{*}{$1.5 \%$} & 12.5 & 6 & 6.5 & 26.2 & Room temperature \\
\hline & 9.5 & 6 & 3.5 & 28.0 & $120^{\circ} \mathrm{C} / 16 \mathrm{~h}$ \\
\hline \multirow{2}{*}{$2.0 \%$} & 13 & 6 & 7 & 25.4 & Room temperature \\
\hline & 10 & 6 & 4 & 28.0 & $120^{\circ} \mathrm{C} / 16 \mathrm{~h}$ \\
\hline
\end{tabular}




\subsection{Form a Semi-Permeable Membrane}

Organic amine inhibitor permeates into the stratum with filtrate, and adsorbs onto the surface of the borehole wall by absorption and electrostatic interaction to reduce the pore space, which is advantageous to the formation of selective semi-permeable membrane and helps to improve the efficiency of formation of rock membrane. Pressure Transmission experiment was used to evaluate the membrane efficiency, and the basic principle is to set up an initial pressure difference on both ends of the cores $\left(\Delta p=p_{\text {up }}-p_{\text {down }}\right)$, and keep $p_{u p}$ unchanged, then test the fluid dynamic pressure changes of the cores at bottom using the pressure sensor and differential pressure sensor. As can be seen from (Fig. 4), PT experiment showed organic amine and complexing aluminum cannot stop the pressure to transmit through the cores respectively, but combination of organic amine and complexing aluminum showed very good effect to slow down the pressure transmission indicating that they formed a membrane on the surface of the cores and it was helpful to prevent drilling fluids to permeate into the formation and advantageous to the stability of borehole wall $[16,17]$.

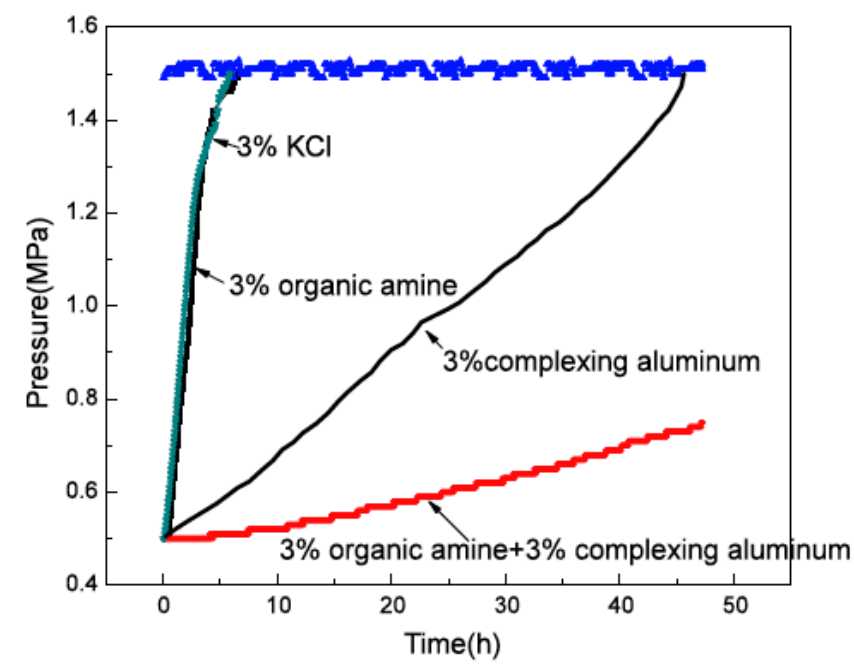

Fig. (4). Experimental results of pore pressure transmission.

\subsection{Reducing the Hydration Interlayer Space of Clay}

The interlayer space of clay, especially sodium montmorillonite, will increase dramatically in the process of surface hydration and osmosis hydration. Low molecular weight organic amine inhibitor will become cationic after protonation. Then the cationic amine group can exchange the hydrated sodium ions and reduce the interlayer space.

As can be seen from Fig. (5), for virgin sodium montmorillonite the interlayer space increased to $2.02 \mathrm{~nm}$ after thorough hydration. When $0.5 \%$ organic amine inhibitor was added into the system, the interlayer space dropped sharply to $1.35 \mathrm{~nm}$. With the addition of organic amine inhibitor, the interlayer space remained almost unchanged, indicating a formation of monolayer adsorption in the gallery of the montmorillonite. Organic amine inhibitor can effectively reduce the interlayer space to the minimum, indicating the best inhibitive effect is obtained $[18,19]$.

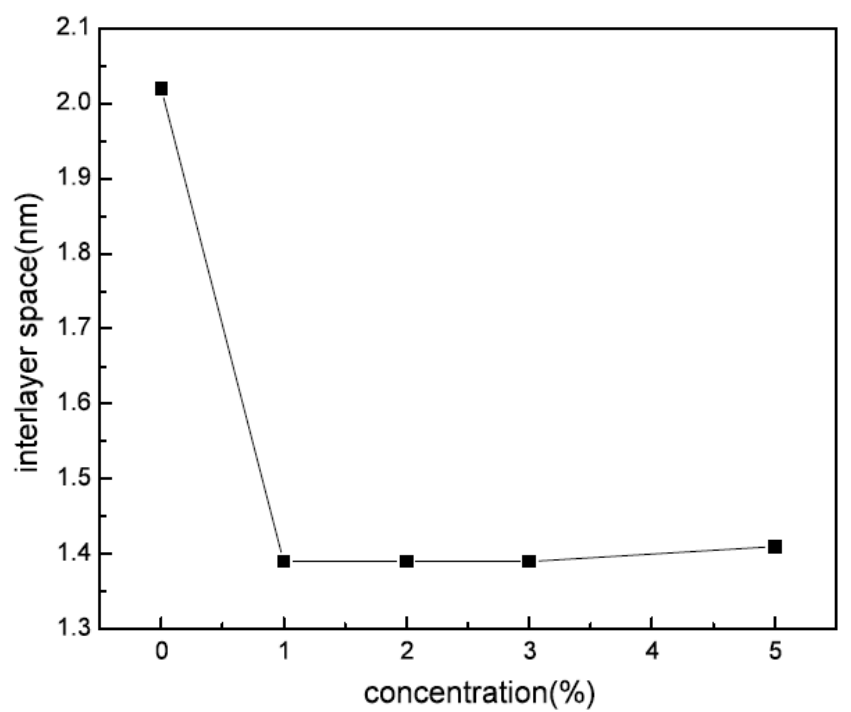

Fig. (5). Variation of interlayer space with addition of organic amine inhibitor.

\section{FIELD APPLICATION OF ORGANIC AMINE IN- HIBITOR}

\subsection{Field Application on Well No.Xin176-Xie12}

Xin176-Xie12 well, a directional well for production in Sha 3 formation, is located in central uplift belt of Dongying sag of Jiyang depression. The designed depth is $3433.78 \mathrm{~m}$. Severe caving was encountered during drilling and cuttings and lumps in this well were found to be soft and were easily dispersed with low fresh water recovery rate. Tripping needed frequent reaming. Due to poor oil and gas show, the operator made decision to sidetrack at $3770 \mathrm{~m}$. To solve the problem, a kind of high performance water-based mud (HPWBM) with organic amine inhibitor and other chemical additives was used and the drilling fluid properties were stable and cutting samples that returned to the shaker were clear without caving blocks. The round trip went smoothly and the drilling was completed successfully at the depth of 3577 [12].

\subsection{Field Application on Liuhua Oilfield}

Liuhua Oilfield is located in the South China Sea which is an environmentally sensitive area. Normally, oil-based drilling fluids is the best choice for an extended reach well with a horizontal displacement of $8000 \mathrm{~m}$, but a kind of high performance water-based mud(HPWBM) with organic amine inhibitor and other chemical additives was successfully used in the extended reach well with a horizontal displacement of $6000 \mathrm{~m}$ in the oilfield. The result showed organic amine exhibited very strong inhibition capability to shale with a low concentrations $(<0.5 \%)$. The primary, secondary and thrice recovery rates were all above $85 \%$ and the rheological and filtration parameters of the drilling fluid with the addition organic amine decreased a little after rolling at $120^{\circ} \mathrm{C}$ for $16 \mathrm{~h}$, which indicated it is suitable for drilling complicated wells and comprehensive analysis showed that HPWBM was a viable water-based fluid alternative that could meet the requirements of drilling extend reach wells and could replace oil-based drilling fluid [20]. 


\section{CONCLUSION}

Organic amine inhibitor has certain effect to decrease the surface tension with little impact on the Zeta potential of the clay. It can effectively inhibit the dispersion of rock cores and cuttings and the persistence is strong which is helpful to the stability of borehole wall and reservoir protection.

The compatibility of organic amine inhibitor with commonly used treatment agents and drilling fluids is well and is conducive to maintain the high temperature stability of the drilling fluids, and has certain effect on drilling fluid filtration performance.

Mechanism of organic amine inhibitor consists of charge neutralization, adsorption, forming a semi-permeable membrane and reducing the hydration interlayer space of clay. They create synergies effect and show the good performance.

Field application of high performance water-based mud (HPWBM) with organic amine inhibitor and other chemical additives were successfully used and indicated organic amine inhibitor has good compatibility with the drilling fluid and maximizes its efficiency.

\section{CONFLICT OF INTEREST}

The authors confirm that this article content has no conflict of interest.

\section{ACKNOWLEDGEMENTS}

Declared none.

\section{REFERENCES}

[1] A. Al-Ansari, K. Yadav, S. Aramco, D. Anderson, R. Leaper, W. Dye, N. Hansen, B. Hughes, Diverse Application of Unique High - Performance Water - Based - Mud Technology in the Middle East. SPE/IADC Middle East Drilling Technology Conference and Exhibition 12-14 September 2005, Dubai, United Arab Emirates, 2005

[2] D. Wang, R. Butler, J. Zhang, "Wettability survey in Bakken Shale using surfactant formulation imbibitions", SPE Reservoir Evaluation \& Engineering, vol. 15, no. 6, pp. 695-705, 2012.

[3] F. Huadi, C. Aldea, B. Mackereth, T. Mukhlis, Successful KClfree, highly inhibitive and cost-effective water-based application, IADC/SPE Asia Pacific Drilling Technology Conference and Exhibition, Ho Chi Minh City, Vietnam, offshore East Kalimantan, Indonesia, 1-3 November 2010.

[4] J. Montilva, E. van Oort, R. Brahim, B. Dye, J. Luzardo, M. McDonald, and L. Quintero, Improved Drilling Performance in Lake Maracaibo Using a Low - Salinity, High - Performance Water - Based Drilling Fluid, American Association for Drilling Engineers; AADE, Houston, AADE 2007.
[5] K. Morton, B. Bomar M. Schiller, J. Gallet, S. Azar, "Selection and Evaluation Criteria for High - Performance Drilling Fluids", SPE Annual Technical Conference and Exhibition, Dallas, Texas 9-12 October 2005.

[6] M.A. Ramirez, S. Benaissa, G. Ragnes, A. Almaraz, Aluminum - Based HPWBM Successfully Replaces Oil - Based Mud To Drill Exploratory Well in the Magellan Strait, Argentina, SPE/IADC Middle East Drilling and Technology Conference, Cairo, Egypt, 22-24 October 2007.

[7] M.A. Ramirez, E. Moura, E. Luna E, and A. Aragao, and H. Taira, HPWBM as a Technical Alternative to Drill Challenging Wells Project: Lessons Learned in Deepwater Brazil, Latin American \& Caribbean Petroleum Engineering Conference, 15-18 April 2007, Buenos Aires, Argentina.

[8] W. Jian-hua, Y. Jie-nian, and D. Tong-wei, "Progresses in the researches on high performance water base muds", Drilling Fluid \& Completion Fluid, vol. 1, p. 1, 2007.

[9] X. Wenfa, X. Xingjin, and W. Changjun, "Study and application of a water-based drilling fluid system containing alcohol ether", Chemical Engineering of Oil and Gas, vol. 4, p. 1, 2004.

[10] Z. Ke-qin, H. Lun, and A. Shu-fang, "An introduction to the high performance water base muds abroad", Drilling Fluid \& Completion Fluid, vol. 3, p. 1, 2007.

[11] Z. Qi-gen, C. Fu, L. Yi, "Development of high performance water base muds abroad", Drilling Fluid \& Completion Fluid, vol. 3, p. 1 , 2007.

[12] L. Kaihe, W. Xuedong, S. Tao, L.R. Kuan, and X.L. Yu, "An highperformance water-based drilling fluid and its application", $A d-$ vanced Materials Research, vol. 476-478, pp. 2304-2310, 2012

[13] S. Xiuchun, L. Hongjun, D. Liyang, S. Dejun, L. He, X. Ruiguo, and R. Zhengjun, "Research on organic amine inhibitor BZ-AN1 used in high inhibitive drilling fluid", Drilling Fluid \& Completion Fluid, vol. 28, no. 2, p. 1, 2011.

[14] L. Benlan, C. Sheng, and S. Xiaodong, "Effects of dispersant on stability of nanosized $\mathrm{Fe}_{3} \mathrm{O}_{4}$ magnetic fluid", Inorganic Chemicals Industry, vol. 8, p. 1, 2011.

[15] M. Songping, C. Ying, L. Xing, "Effects of surfactants on dispersion of Titania Nano fluids", Materials Review, vol. 1, p. 1, 2013.

[16] Z. Shifeng, Q. Zhengsong, H. Wei'an, Z. Hanyi, M. Meng, "Study on high performance aluminum-polyamine based blocking antisloughing drilling fluid", Journal of Southwest Petroleum University (Science \& Technology Edition), vol. 35, no. 4, pp. 159-164, 2013.

[17] Z. Han-yi, Q. Zheng-song, and H. Wei-an, "Properties evaluation and application of polyamine high performance water-based drilling fluid", Science Technology and Engineering, vol. 1, p. 1, 2013.

[18] J. Zhang, J. Chen, and C. Edwards, Lab NMR Study on Adsorption/Condensation of Hydrocarbon in Smectite Clay, SPE/EAGE European Unconventional Resources Conference and Exhibition, Vienna, Austria, 20-22 March 2012.

[19] L. Wei, P. Xiaolin, and O. Yangwei, "Research on highperformance aluminium conpound drilling fluid system", Drilling Fluid \& Completion Fluid. vol. 7, p. 1, 2011.

[20] Y. Jienian, G. Jiaojiao, L. Zhiyong, G. Deli, W. Jianhua, and Z. Shengying, Design of Water-based Drilling Fluids for an Extended Reach Well with a Horizontal Displacement of $8000 \mathrm{~m}$ Located in Liuhua Oilfield, International Oil and Gas Conference and Exhibition in China, Beijing, China, 8-10 June 2010.

(C) Lv et al.; Licensee Bentham Open.

This is an open access article licensed under the terms of the Creative Commons Attribution Non-Commercial License (http://creativecommons.org/licenses/by-nc/3.0/) which permits unrestricted, non-commercial use, distribution and reproduction in any medium, provided the work is properly cited. 